\title{
Probing Reaction Dynamics of Transition-Metal Complexes in Solution via Time-Resolved Soft X-ray Spectroscopy
}

\author{
Nils Huse ${ }^{1}$, Tae Kyu Kim², Munira Khalil ${ }^{3}$, Lindsey Jamula ${ }^{4}$, \\ James K. McCusker ${ }^{4}$, and Robert W. Schoenlein ${ }^{1}$ \\ ${ }^{1}$ Chemical Sciences Division, Lawrence Berkeley National Laboratory, 1Cyclotron Road, Berkeley, CA 94720, \\ USA \\ E-mail: nhuse@lbl.gov \\ ${ }^{2}$ Department of Chemistry, Pusan National University, Geumjeong-gu, Busan 609-735, Korea \\ ${ }^{3}$ Department of Chemistry, University of Washington, Seattle, WA 98195, USA \\ ${ }^{4}$ Department of Chemistry, Michigan State University, East Lansing, MI 48824, USA
}

\begin{abstract}
We report the first time-resolved soft x-ray measurements of solvated transition-metal complexes. Ledge spectroscopy directly probes dynamic changes in ligand-field splitting of $3 d$ orbitals associated with the spin transition, and mediated by changes in ligand-bonding.
\end{abstract}

\section{Introduction}

A major goal in chemistry is to quantitatively probe chemical bonding dynamics at the molecular level. That is: to follow valence-charge distributions in real time, to observe the formation and dissolution of chemical bonds, and to understand their cooperative relationship with atomic rearrangement and the formation of new molecular structures. The emerging technique of ultrafast soft $\mathrm{x}$-ray spectroscopy provides important new insight to molecular dynamics because $\mathrm{x}$-ray corelevel transitions (e.g. $2 \mathrm{p}_{1 / 2} \rightarrow$ LUMO) are element specific, symmetry and spin state selective, and effective for probing lighter elements $(\mathrm{C}, \mathrm{N}, \mathrm{O}$ etc.) that are biologically relevant. Thus, the $\mathrm{X}$-ray near-edge spectral region (XANES) provides a quantitative fingerprint of the local chemical bonding geometry (orbital hybridization etc.) in the vicinity of the atom(s) of interest. This is particularly powerful for understanding molecular dynamics in solution, where much important chemistry occurs, and where the solvent environment substantially influences reaction dynamics (often precluding quantitative interpretation

of transient absorption spectra in the visible regime). A significant experimental challenge for soft $\mathrm{x}$ ray spectroscopy in liquid-phase has been the requirement for sub-micron liquid films (due to large absorption cross-sections) that remain stable in a vacuum environment under typical photo-excitation conditions.

Here we report the first time-resolved Fe L-edge spectroscopy of the ultrafast photo-induced intersystem crossing occurring in the solvated transition-metal complex $\left[\mathrm{Fe}\left(\operatorname{tren}(\mathrm{py})_{3}\right)\right]^{2+}$. Interest in the $\mathrm{Fe}^{\mathrm{II}}$ complex stems from the rapid spin transition $(\Delta S=2)$ that is thought to result from changes in the ligand-field splitting of the Fe- $3 d$ orbitals, and associated changes in $\mathrm{Fe}-3 d / \mathrm{N}-2 p$ hybridization. These changes are mediated by a dilation of the octahedrally-coordinated Fe- $\mathrm{N}$ bonds, and optically triggered by the initial metal-to-ligand charge transfer excitation [1-3]. Time-resolved measurements at the Fe L-edges directly probe the evolution of the ligand-field split $3 d$ orbitals via selective transitions from the spin-orbit-split Fe- $2 p_{1 / 2} / 2 p_{3 / 2}$ levels.

The $\left[\mathrm{Fe}\left(\operatorname{tren}(\mathrm{py})_{3}\right)\right]^{2+}$ complex in Fig. 1A has an octahedral ligand geometry around a central $\mathrm{Fe}$ atom [1]. Hybridization with the nearest ligand $\mathrm{N}-2 p$ orbitals lifts the degeneracy of the Fe-3d orbitals to form $t_{2 g}$ and $e_{g}$ levels as pictured schematically in Fig. 1C. The associated Fe- $\mathrm{N}$ charge-transfer transition has a maximum absorption at 560nm. Optical spectroscopy has extracted a 300fs time constant, and this has been interpreted as a signature of intersystem crossing to a high-spin state with a change in spin of $\Delta \mathrm{S}=2$, accompanied by an $\sim 0.2 \AA$ elongation in the Fe-N bond distance and an 
octahedral distortion as revealed by hard x-ray (EXAFS) spectroscopy [1-3]. The ultrafast intersystem crossing and the structural changes point to an intricate interplay of electronic, atomic, and magnetic structure that is yet to be deciphered.
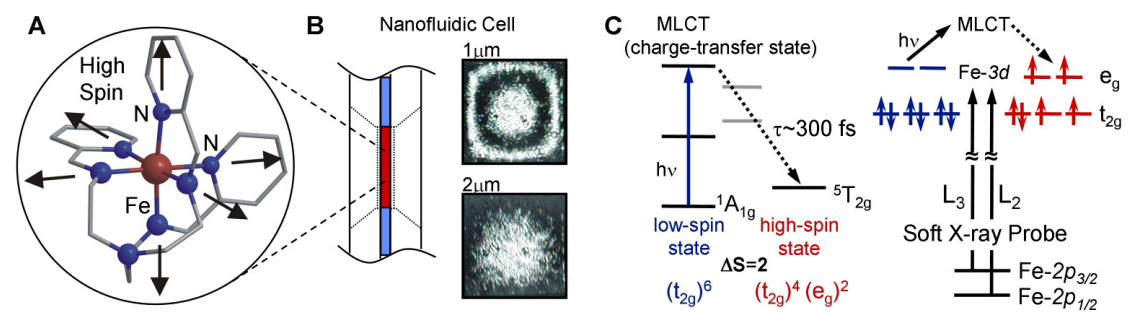

Fig. 1. Schematic of $\left[\mathrm{Fe}\left(\operatorname{tren}(\mathrm{py})_{3}\right)\right]^{2+}$, arrows indicate the metal-ligand bond change associated with the spin transition (A). Nanofluidic cell layout and transmission interferograms that indicate liquid film thickness (B). Simplified energy levels of intersystem crossing after 560nm excitation and L-edge spectroscopy of the $\mathrm{Fe}^{\mathrm{II}}$ complex with soft $\mathrm{x}$-ray probe pulses $(\mathbf{C})$.

\section{Experimental Methods}

Soft x-ray spectroscopy experiments are made possible by a novel nanofluidic cell design (Fig. 1B) in which a liquid film is held between two $100 \mathrm{~nm}$ silicon nitride membranes. The interior cell pressure is balanced against the pressure of the sample chamber, thereby controlling the film thickness with sub$200 \mathrm{~nm}$ accuracy [4]. A third silicon-nitride membrane isolates the low-pressure sample chamber from the ultrahigh vacuum of the femtosecond soft x-ray beamline at the Advanced Light Source. The Fe ${ }^{\mathrm{II}}$ complex is dissolved in acetonitrile at $100 \mathrm{mM}$ concentration, and excited with ultrashort $560 \mathrm{~nm}$ pulses at $1 \mathrm{kHz}$. Transient absorption changes at the $\mathrm{Fe} \mathrm{L}_{2}$ and $\mathrm{L}_{3}$ edges are probed with tunable 70ps synchrotron pulses at $2 \mathrm{kHz}$ as a function of $\mathrm{x}$-ray probe energy and time delay between excitation and probe pulses. From the spectral measurements of both the ground-state and photo-excited sample, transient $\mathrm{x}$-ray absorption spectra of the high-spin state at the $\mathrm{Fe} \mathrm{L}_{2}$ and $\mathrm{L}_{3}$ edges are reconstructed.

\section{Results and Discussion}

Figure 2A shows the absorption of the high and low-spin state after the photo-induced metal-to-ligand charge transfer. These are to our knowledge the first time-resolved solution-phase transmission spectra ever recorded in the soft $\mathrm{x}$-ray region. The $\mathrm{L}_{3}$ edge of the high-spin state shows a pronounced red-shift by $1.6 \mathrm{eV}$ with no discernable change in linewidth relative to the low-spin state. However, more complex absorption features on the blue side of the maximum are present. The $\mathrm{L}_{2}$ edge exhibits only a small red-shift by about $0.4 \mathrm{eV}$ upon laser excitation but the absorption cross-section is diminished by nearly a factor of 2 . The time delay scans in Fig. 2B show changes in sample absorption at specific probe photon energies, consistent with the spectral scans. While the absorption decreases at the spectral absorption maxima of the low-spin state after excitation, it increases around $707 \mathrm{eV}$ where the absorption of the high-spin state is maximal. The delay scans reflect the 70ps width of the x-ray probe pulses and demonstrate that the $\mathrm{Fe}^{\mathrm{II}}$ compound reaches its meta-stable high-spin state within the time resolution of the experiment. Differential spectra at 5 ns delay demonstrate that the evolution of the high-spin electronic structure is complete within 70ps.

Our time-resolved liquid-phase measurements are consistent with static x-ray measurements of a similar compound in crystalline phase [5] (where the high-spin state is trapped at low temperature), and offer an important opportunity to investigate the solvent influence on the ultrafast dynamics of the electronic and spin states. Our experimental results are in good agreement with theoretical calculations of the high- and low-spin electronic structure [5]. In particular, the size of the red-shift of both $\mathrm{L}_{3}$ and $\mathrm{L}_{2}$-edges is reproduced. Crystal field multiplet calculations attribute our observations to changes in $3 d$ occupancy. The red-shift of the $\mathrm{L}_{3}$ edge is caused by a change in ligand-field splitting 
of $0.5 \mathrm{eV}$ and a core-hole shift of about $1 \mathrm{eV}$. The red-shift of the $\mathrm{L}_{2}$ edge seems to directly reflect the change in ligand-field splitting.
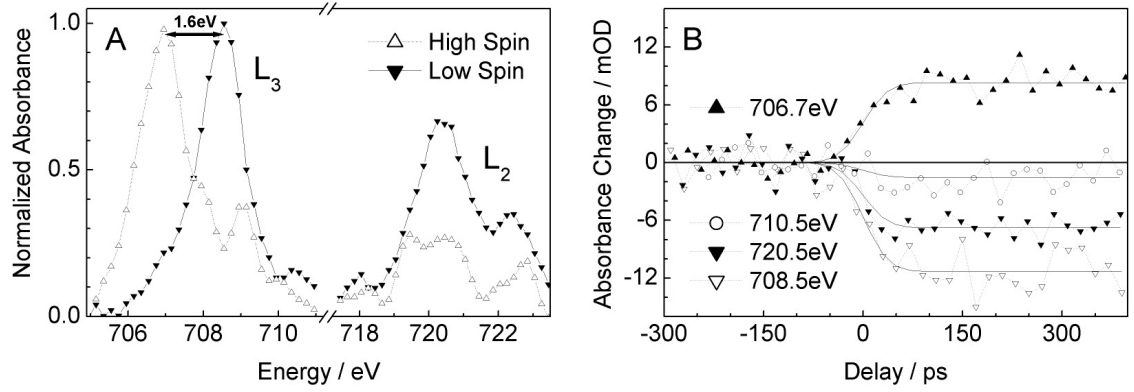

Fig. 2. $\mathrm{L}_{2}$ and $\mathrm{L}_{3}$ absorption spectra of the initial low spin complex, and the transient photo-induced high-spin complex at 150ps delay (A). Sample absorption change as a function of delay between the 560nm excitation pulse and the soft x-ray probe pulse at specific x-ray energies. Solid lines are fits to the data based on an instantaneous absorption change convolved with a 70ps pulse, with negligible relaxation within the measurement window $(\mathbf{B})$.

\section{Conclusions}

We report the first time-resolved soft x-ray spectroscopy of solution-phase molecular dynamics. Changes in ligand-field splitting and spin-state populations in $3 d$ orbitals of the $\mathrm{Fe}^{\mathrm{II}}$ complex are directly probed via transient absorption changes of the $\mathrm{Fe} \mathrm{L}_{2}$ and $\mathrm{L}_{3}$ edges following photo-induced metal-to-ligand charge transfer. With the emergence of high-flux ultrafast soft x-ray sources, details on interplay between atomic structure, electronic states, and spin contributions will be revealed. Our experimental approach opens the door to femtosecond soft x-ray investigations of liquid phase chemistry that have previously been inaccessible.

Acknowledgements. This work was supported by the Department of Energy under Contract No. DEAC02-05CH11231.

Legal Disclaimer. This document was prepared as an account of work sponsored by the United States Government. While this document is believed to contain correct information, neither the United States Government nor any agency thereof, nor The Regents of the University of California, nor any of their employees, makes any warranty, express or implied, or assumes any legal responsibility for the accuracy, completeness, or usefulness of any information, apparatus, product, or process disclosed, or represents that its use would not infringe privately owned rights. Reference herein to any specific commercial product, process, or service by its trade name, trademark, manufacturer, or otherwise, does not necessarily constitute or imply its endorsement, recommendation, or favoring by the United States Government or any agency thereof, or The Regents of the University of California. The views and opinions of authors expressed herein do not necessarily state or reflect those of the United States Government or any agency thereof or The Regents of the University of California.

1 J. E. Monat and J. M. McCusker, J. Am. Chem. Soc. 122, 4092, 2000.

2 M. Khalil, M. A. Marcus, A. L. Smeigh, J. K. McCusker, H. H. W. Chong, and R. W. Schoenlein, J. Phys. Chem. A 110, 38, 2006.

3 W. Gawelda, V.-T. Pham, M. Benfatto, et al., Phys. Rev. Lett. 98, 057401, 2007.

4 D. Kraemer, M. L. Cowan, A. Paarmann, N. Huse, E. T. J. Nibbering, T. Elsaesser, and R. J. D. Miller, Proc. Nat. Acad. Sci. 105, 437, 2008.

5 V. Briois, Ch. Cartier dit Moulin, J Ph. Sainctavit, Ch. Brouder, and A.-M. Flank, J. Am. Chem. Soc. 117, 10191995. 